\title{
Multiple polariton modes originating from the coupling of quantum wells in planar microcavity
}

\author{
C. Ouellet-Plamondon,* G. Sallen, F. Jabeen, D. Y. Oberli, and B. Deveaud \\ Institute of Condensed Matter Physics, École Polytechnique Fédérale de Lausanne (EPFL), CH-1015 Lausanne, Switzerland
}

(Received 28 April 2015; published 31 August 2015)

\begin{abstract}
We report on the observation of multiple polariton modes, originating from an electronic coupling between quantum wells inside a planar microcavity. A series of excitonic transitions are measured for a bare quantum well stack and are precisely identified to electron-hole transitions originating from the coupling of the wells. When the quantum well stacks are placed at the antinode of a high $Q$-factor microcavity, a series of anticrossings is observed, which is characteristic of a multiplicity of polariton modes. This behavior is simulated using a coupled oscillator model accounting for the cavity mode and all allowed excitonic transitions.
\end{abstract}

DOI: 10.1103/PhysRevB.92.075313

PACS number(s): 78.55.Cr, 78.67.De, 78.67.Pt

\section{INTRODUCTION}

An exciton-polariton is a light-matter quasiparticle arising from the strong coupling of an exciton and a photon [1]. The study of such elementary excitation is usually achieved by placing a quantum well (QW) at an antinode of the electromagnetic field of a planar microcavity [2]. Microcavity polaritons have been shown to exhibit out of equilibrium Bose-Einstein condensation [3-6] when the system is excited nonresonantly. In order to achieve polariton BEC, two conditions must be fulfilled. First, the coupling strength must be high enough in order to maintain the strong coupling at high density $[7,8]$, and second, the polariton critical density must be reached before the onset of the excitonic Mott transition, where the system is no longer bosonic due to the nature of the electron-hole plasma [9]. In terms of sample design, these two conditions can be met in principle if the number of QWs in the microcavity is increased because it increases the coupling strength but also decreases the exciton density per QW.

In this work, we show that placing multiple InGaAs/GaAs QWs inside a microcavity does not increase the coupling strength following the square root of the number of QWs $\sqrt{N_{\mathrm{QW}}}$ as expected but instead gives rise to multiple polariton modes. The small height of the tunneling barrier created by the low indium content of these QWs (an order of magnitude less than GaAs/AlAs QW) causes the exciton level of individual wells to hybridize [10-12], creating a series of excitonic transitions.

Multimode polaritons have been measured in a variety of sample designs where the coupling occurs between either a single exciton state in a multimode cavity or the opposite. In the first case, it was demonstrated with coupled planar microcavities [13-16] or by confining the optical mode in more than one dimension [5,17]. The coupling of a single cavity mode to many exciton states has been achieved through the monolayer thickness variation of GaAs/AlAs QWs [18], with QWs of different thicknesses [19] or when coupling to the charged exciton transition [20,21].

InGaAs QWs have been frequently used to study the exciton-polariton, either for a single QW microcavity $[16,17,22-24]$ or QW stacks with varying indium content $[8,25-30]$. In the later, the multimode polariton was not

*clauderic.ouellet-plamondon@epfl.ch observed but, in almost every case, an additional emission peak between the lower and upper polariton branch was present in the spectra. This transition was assigned to an uncoupled exciton transition [26,29] as a consequence of the quality of the samples with an inhomogeneously broadened transition. In the present study, we show that, with a high quality sample, these additional transitions are effectively coupled with the cavity resulting in strong coupling with up to seven excitonic transitions.

\section{SAMPLES AND EXPERIMENTAL DETAILS}

Two samples were grown by molecular-beam epitaxy (MBE) to study the effect of interwell coupling in a microcavity. The first one consists of a single stack of three $12 \mathrm{~nm}$ $\mathrm{In}_{0.03} \mathrm{Ga}_{0.97} \mathrm{As} \mathrm{QWs}$ with a spacer of $10 \mathrm{~nm}$. The low indium content is purposely chosen to create a low tunneling barrier between the QWs in order to obtain electron and hole wave functions that extend over the whole QW stack. The second sample is a microcavity consisting of 20(23) top(bottom) AlAs/GaAs distributed Bragg reflector (DBR) pairs with a $2 \lambda$ spacer where $\lambda$ is the cavity resonance wavelength. Three stacks of three QWs (identical to the first sample) are placed at the antinode of the electromagnetic field inside the cavity. The microcavity is grown with a wedge along the radius of the wafer allowing one to vary the exciton-cavity detuning when probing different sample positions. The quality factor of the cavity is around $Q \cong 27000$, measured by scanning a narrow tunable laser across the cavity resonance [full width at half maximum $(\mathrm{FWHM})=56 \mu \mathrm{eV}]$ at negative cavity detuning. For this sample, we cannot properly define the vacuum Rabi splitting as for the case of a single exciton resonance since the coupling occurs with up to seven excitonic transitions [see Eq. (1) and Fig. 4].

Photoluminescence (PL) studies were carried using a standard confocal configuration exciting with a HeNe laser at low excitation power $(P=100 \mu \mathrm{W})$ and a spot size of $\sim 25 \mu \mathrm{m}$. A diaphragm inside the confocal microscope objective allows selecting only the emission from $k=0$ (polariton with zero in-plane wavevector). The samples were cooled to $10 \mathrm{~K}$ using a closed circuit He cryostat. Photoluminescence excitation (PLE) was performed in transmission geometry, exciting with a cw Ti:sapphire laser $(P=10 \mu \mathrm{W})$ and a spot size of $\sim 25 \mu \mathrm{m}$. For these measurements, the sample was cooled to liquid helium temperature $(\sim 4 \mathrm{~K})$ using a cold finger cryostat. 


\section{QUANTUM WELL STACK}

Figure 1 shows the PL spectrum of the bare stack of QWs as well as the PLE when detecting on the energy of the lowest exciton line (1.4894 eV). One clearly sees a sharp emission peak and additional structures at higher energy. The free exciton line from the GaAs is largely suppressed in PL, suggesting an efficient carrier capture to the QWs. The inset shows the PL when exciting below the GaAs bandgap, at an energy of $1.5 \mathrm{eV}$, indicating that the dominant peak measured in PL is composed of two transitions separated by $0.39 \pm 0.05 \mathrm{meV}$. This structure was further confirmed by fitting the spectrum using a Lorentzian profile (not shown). A total of six excitonic transitions can be identified from the PL, with linewidths ranging from 0.4 to $0.6 \mathrm{meV}$ for the low energy doublet and 1 to $2.6 \mathrm{meV}$ for the others, demonstrating the high quality of the sample. When comparing the PL and PLE, we confirm that for each PL transition, there is a resonance in the PLE signal, indicating these are sharp excitonic transitions. Although the resonance in PLE at a relative energy of $5.7 \mathrm{meV}$ is not clearly seen in PL, it is still needed to obtain a proper Lorentzian fit of the entire spectrum.

In order to determine the origin of the measured excitonic transitions, we calculate the electron-hole confined states of the QW structure using Nextnano, which is commonly used for semiconductor simulation [31]. The system of three QWs is solved using the effective mass approximation to obtain the wave function and energy of electron and hole states confined to the QW stack. Because of the small lattice mismatch between GaAs and InGaAs, strain has to be included to properly simulate the structure. As a result, heavy holes are confined while the light holes are not, as it is known from earlier studies with InGaAs QWs [10,32].

In Fig. 2, we present the resulting envelope wave functions (not to scale) of confined electron and hole states for a stack of three $\operatorname{In}_{0.03} \mathrm{Ga}_{0.97} \mathrm{As}$ QWs. Three electron states are confined in the QW stack whereas the calculation is limited to five states

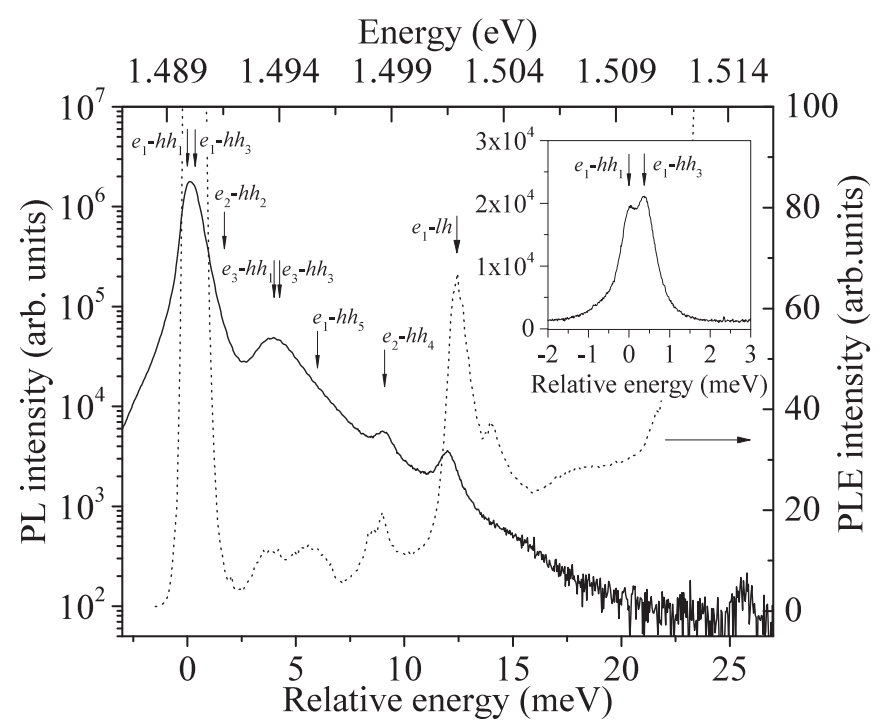

FIG. 1. PL spectrum of a stack of three $12 \mathrm{~nm} \mathrm{In}_{0.03} \mathrm{Ga}_{0.97}$ As QWs with a $10 \mathrm{~nm}$ spacer and the corresponding PLE when detecting at the energy of the lowest exciton transition. The inset shows the PL when the excitation laser is at an energy of $1.5 \mathrm{eV}$.
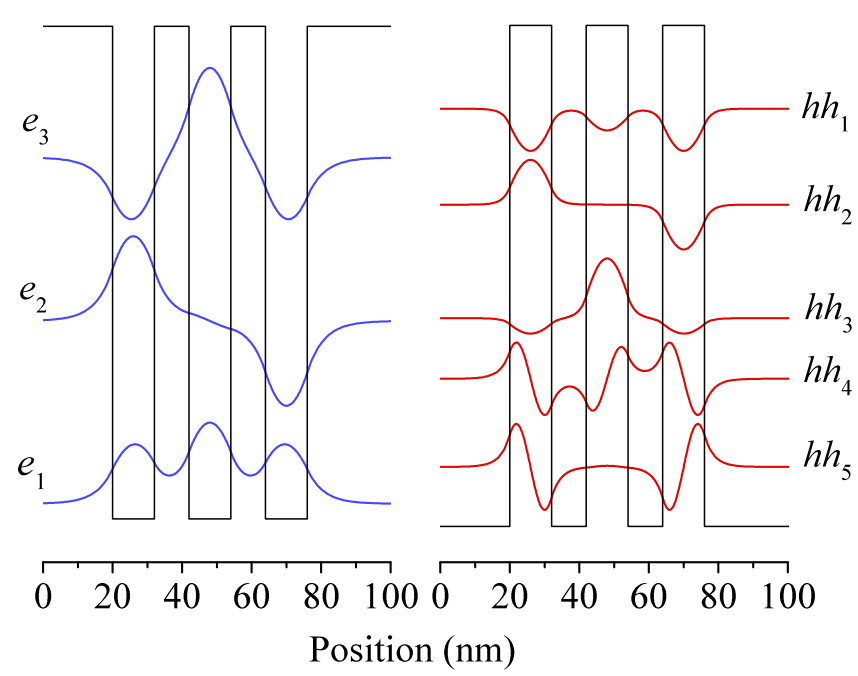

FIG. 2. (Color online) Calculated wave functions for bound electron $e_{i}$ and heavy hole $h h_{i}$ states. The black lines show schematically the positions of the potential barriers. An offset between wave functions is introduced to distinguish different states and the amplitude is not to scale. The integer $i$ indicates an increase (decrease) in energy for electrons (holes) and corresponds to $(i-1)$ nodes of the eigenfunction.

for the heavy holes (additional hole states can be calculated but give either an exciton transition too high in energy or with a negligible oscillator strength). We evaluate the transition probability amplitude between the electron and hole states using these wave functions through the dipole matrix element [33]: $\langle i|A \cdot p| f\rangle \propto\left\langle F_{c}|A \cdot p| F_{v}\right\rangle \int d z \chi_{e}^{*}(z) \chi_{h h}(z)$, where $A$ is the vector potential of the electromagnetic field, $p$ is the electron momentum operator, $\chi_{e}(z), \chi_{h h}(z)$ are the electron and hole envelope wave functions along the confinement direction and $\left|F_{c, v}\right\rangle=f_{c, v}(r)\left|u_{c, v}\right\rangle$, is the product of the in-plane wave function and of the Bloch wave function for the conduction and valence band. If one assumes identical dipole moments and in-plane wave functions for all transitions, the relative transition probability is simply evaluated through the overlap integral squared between $\chi_{e}$ and $\chi_{h h}$. When comparing the wave functions from Fig. 2, we notice that the integral is nonzero only for electron and hole states of same parity, which sets a strict selection rule for the allowed transitions. The energy of the excitonic transitions are obtained by taking the energy difference between the electron and hole state, without considering possible corrections due to Coulomb interaction (changes in the exciton binding energy).

Results from the calculation are shown in Fig. 3, where we plot the calculated and measured transition energies relative to their respective lowest transition, labeled $E_{e 1-h h 1}$. The calculation accurately reproduces the energy separation of the measured excitonic transitions with a quadratic error of $\Delta E_{c \mathrm{QW}}=1.62 \mathrm{meV}$. This confirms that all measured transitions are associated to excitons arising from interwell coupling. Therefore, we can use the calculation to label each of the measured transitions using the electron and hole index as in Table I. Two transitions, namely, $e_{2}-h h_{2}$ and $e_{3}-h h_{1}$, are not resolved in the PL spectrum but cause a broadening of the transitions at $\Delta E_{e 1-h h 3}=0.35$ and $\Delta E_{e 3-h h 3}=3.9 \mathrm{meV}$, 


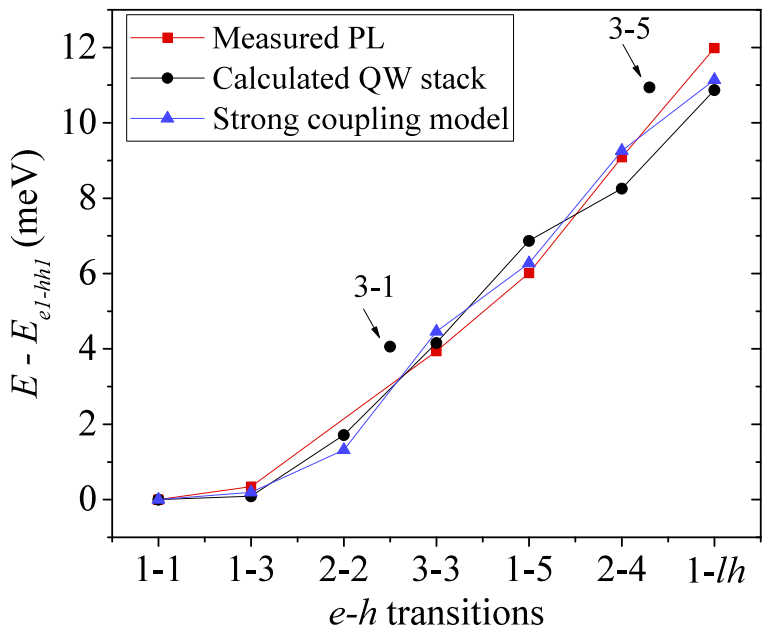

FIG. 3. (Color online) Energy of electron-hole transitions relative to the first transition $E_{e 1-h h 1}$ for the bare QW sample (red), calculated for a single QW stack (black), and the relative values used for the strong coupling model (blue). The relative value for the $e_{3}-h h_{1}$ and $e_{3}-h h_{5}$ are shown for comparison. The label 1-lh indicate the transition from $e_{1}$ to the light hole.

respectively. From the calculation, the transition situated at $11 \mathrm{meV}$ from the ground state can be assigned to the $e_{3}-h h_{5}$ transition, to a transition between the first electron and the unconfined light hole bound by Coulomb interaction, or to a light hole confined inside the barrier in between wells. The comparison with the PLE clearly shows a stronger resonance compared to the other transitions, indicating that this transition corresponds to the light-hole one. PL from a single QW also supports this identification [34].

\section{MULTIMODE POLARITON}

In the following, we study the microcavity sample to evidence the effect of the additional exciton states on the coupling to the cavity field. The PL intensity is shown as a function of the position on the sample in Fig. 4(a) and the spectrum for specific positions in Fig. 4(b) as indicated by dashed lines in Fig. 4(a). As the cavity mode gets closer to the exciton resonances from the low energy side, the exciton states are blueshifted in energy, the maximum shift being fixed by the energy difference between the two neighboring states. Such a

TABLE I. Calculated relative energy and light-matter coupling strength for electron-hole transition. The last column shows the values of the normalized integral used for the manual fit. The values in parentheses indicate the values before normalization.

\begin{tabular}{lccc}
\hline \hline$e-h$ transitions & $\Delta \mathrm{E}(\mathrm{meV})$ & $\Omega_{i j} / \Omega_{0}$ & Manual fit \\
\hline$e_{1}-h h_{1}$ & 0 & $0.96(0.83)$ & $0.95(0.81)$ \\
$e_{1}-h h_{3}$ & 0.09 & $0.23(0.20)$ & $0.62(0.53)$ \\
$e_{2}-h h_{2}$ & 1.72 & $0.88(0.76)$ & $1.00(0.85)$ \\
$e_{3}-h h_{1}$ & 4.07 & $0.02(0.01)$ & $0.02(0.01)$ \\
$e_{3}-h h_{3}$ & 4.16 & $1.00(0.87)$ & $0.39(0.33)$ \\
$e_{1}-h h_{5}$ & 6.87 & $0.13(0.11)$ & $0.39(0.33)$ \\
$e_{2}-h h_{4}$ & 8.26 & $0.16(0.14)$ & $0.31(0.27)$ \\
$e_{1}-l h$ & 10.87 & $0.07(0.06)$ & $0.18(0.15)$ \\
\hline \hline
\end{tabular}
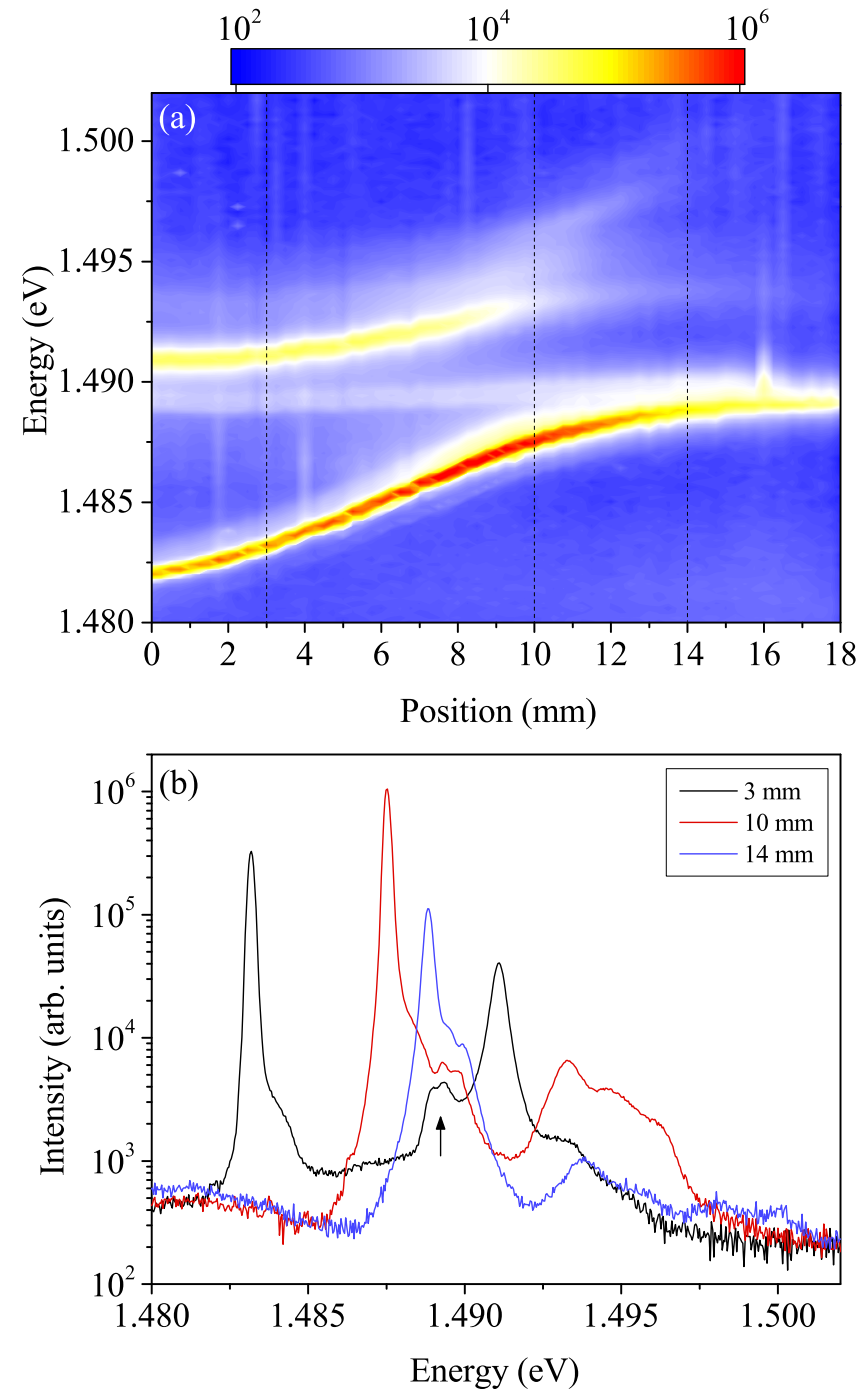

FIG. 4. (Color online) (a) PL intensity map in log scale as a function of the position on the microcavity sample. (b) PL spectra for three positions on the sample as indicated by the dashed lines in (a). The arrow indicates the doublet of peaks at $1.489 \mathrm{eV}$.

behavior is the main characteristic of strongly coupled states. Moreover, when comparing the spectra in Fig. 4(b), we clearly see that the two transitions initially at around $1.489 \mathrm{eV}$ are displaced to higher energies as they get in resonance with the cavity. Although this energy shift is small $(450-550 \mu \mathrm{eV})$, it can only be explained by an interaction with the cavity for the following reasons. As evidenced in Fig. 4(b), the blueshift of the low energy transition of this doublet stops once its energy reaches the initial value of the second doublet transition. Alternatively, the small energy shift could be assigned to a monolayer variation of the QWs thickness [35]; the measured shift would, however, be more than twice the calculated value if each QW in the stack were thinned by one monolayer $(\sim 250 \mu \mathrm{eV})$, which rules out this possibility. Furthermore, a monolayer variation of one or more $\mathrm{QW}$ within the stack would also imply a breaking of the symmetry of the stack, hence additional excitonic transitions would be allowed while none are observed within our resolution $(\sim 150 \mu \mathrm{eV})$. From these observations, we conclude that all measured exciton states are 
strongly coupled with a single cavity mode. Finally, the high energy shoulder present on the lower polariton branch in the spectra is due to our finite resolution in $k$, hence, emission at low $k$ from the polariton dispersion. The linewidth of the lower polariton mode at negative cavity detuning is $\sim 150 \mu \mathrm{eV}$ when measured on a higher resolution setup.

We use the following light-matter interaction Hamiltonian to model our data, with $\hbar=1$ :

$$
H=\omega_{c} b^{\dagger} b+\sum_{i, j} E_{i j} a_{i j}^{\dagger} a_{i j}+\sum_{i, j} \frac{\Omega_{i j}}{2}\left(a_{i j}^{\dagger} b+b^{\dagger} a_{i j}\right),
$$

where the $i, j$ indices correspond to the electron and hole state numbers, $\hbar \omega_{c}$ is the cavity mode energy, $E_{i j}$ is the exciton energy, and $a^{\dagger}(a), b^{\dagger}(b)$ are creation (annihilation) operators for the exciton and cavity photon. The allowed excitonic transitions used for the sum are shown in Table I. This Hamiltonian has been successfully used to simulate coupling from inhomogeneously broadened excitonic transitions $[18,26]$ where the coupling strength is assumed to be constant for all excitonic subtransitions. For the case of coupled QWs, the light-matter coupling strength is evaluated for each transition through the overlap between the electron and hole wave function and the electromagnetic field inside the cavity [36,37]:

$$
\Omega_{i j}=\frac{\Omega_{0}}{I_{M}} \int \chi_{h_{j}}^{*}(z) E(z) \chi_{e_{i}}(z) d z,
$$

where $\chi_{e_{i}}, \chi_{h_{j}}$ are the $i$ th electron and $j$ th hole wave function as calculated above and $I_{M}$ is the integral between the states $i-j$ having the highest overlap. Assuming the same dipole strength for all transitions and normalizing the integrals to $I_{M}$, the coupling strengths between the cavity and all transitions can be fitted using a single parameter $\Omega_{0}$, which would be the vacuum Rabi splitting for the coupling of a cavity with a single exciton state. The Hamiltonian is diagonalized in order to obtain the polariton energy as a function of position on the sample (cavity detuning). For the calculation, we use a single QW stack at the antinode of a $\lambda$ cavity; the number of stacks is considered simply by increasing the coupling strength by a factor $\sqrt{3}$. [26]

Results from such calculations are shown in Fig. 5(a) where the dots represent the energy position of each polariton line measured as seen in Fig. 4 and the gray region surrounding them corresponds to the error bar. The full red lines show the best fits obtained after diagonalizing Hamiltonian (1) for a coupling parameter of $\Omega_{0}=4.0 \pm 0.1 \mathrm{meV}$ and the values of the overlap integral are given in Table I. Because the light-hole wave function in the continuum is not known, we use the value of overlap of the state $e_{3}-h h_{5}$ due to its similar relative energy. The quadratic error from fitting all polariton transitions in this case is $\Delta E_{1}=8.17 \pm 0.05 \mathrm{meV}$, which is quite low considering the number of data points. The straight dashed lines are the uncoupled excitonic transitions. As the binding energy is not precisely known and as some transitions are measured in the microcavity sample but not in the bare QW, we allow a slight energy variation for each transition in order to improve the fit. The relative values used are shown in Fig. 3 confirming their validity with respect to the measured bare QW excitonic transitions. The $e_{3}-h h_{1}$ transition is not included in the fitting procedure because of its relatively small overlap
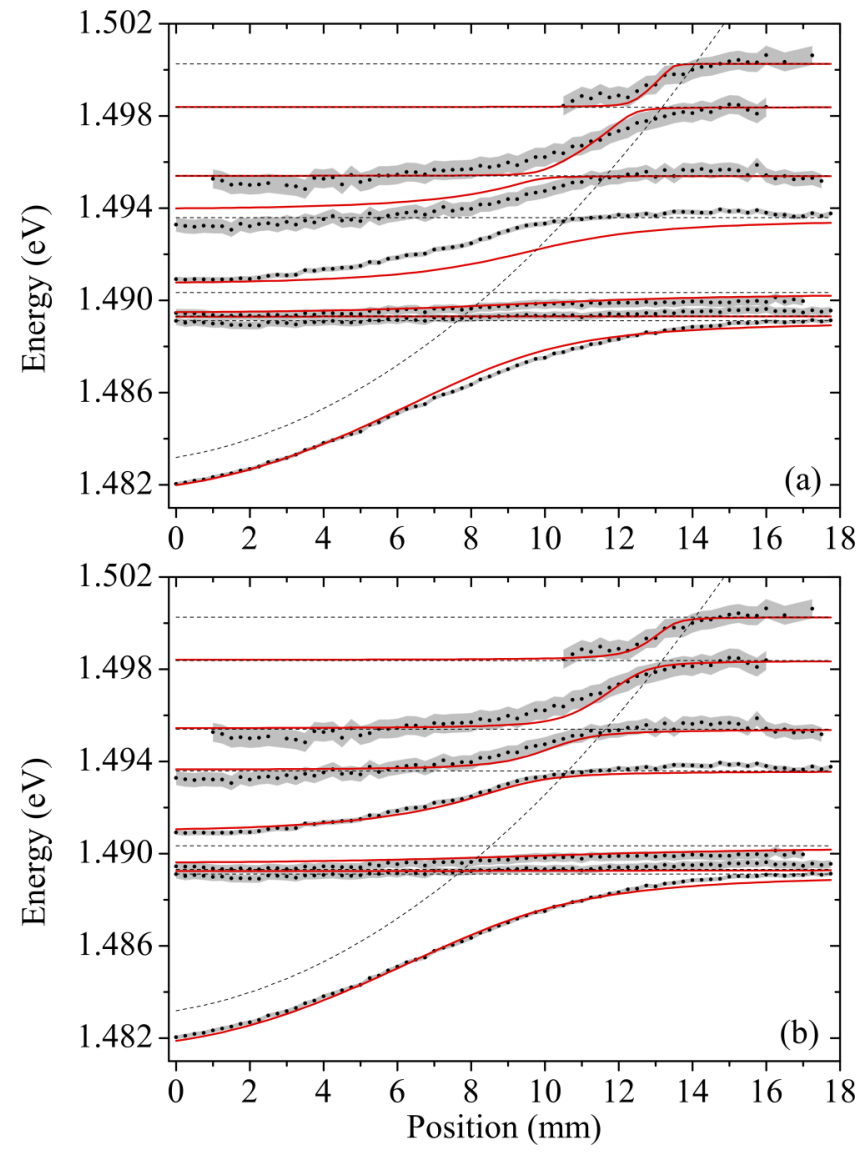

FIG. 5. (Color online) Polariton energy as a function of sample position. The dots show the extracted peak energy and the gray regions show the uncertainty for each state. The red full lines shows the best fits using Eqs. (1) and (2) in (a) or by manually adjusting the overlap parameter in (b). The dashed lines represent the energy of the cavity mode (parabola) and exciton transitions (straight). In both cases, the coupling parameter is $\Omega_{0}=4 \mathrm{meV}$ and the values of the the overlap integrals are given in Table I.

compared to the other transitions. The cavity mode shown by the curved dashed line was determined by PL using a pump laser diode $(\lambda=660 \mathrm{~nm})$ at high power to reach the lasing threshold of vertical cavity surface emitting laser (VCSEL) in order to precisely extract the cavity mode. The emission energy was measured at different positions on the sample just after the threshold occurred to limit any possible multimode behavior from the cavity. Since the cavity shows a perfect parabolic variation, only the fit is shown in Fig. 5.

\section{DISCUSSION}

The difference of the relative coupling strength of each transition (Table I) can be explained qualitatively by comparing the wave functions displayed in Fig. 2. From the figure, it is clear that the $e_{i}-h h_{j}$ transitions have a smaller coupling compared to the $e_{i}-h h_{i}$ ones because the initial and final state do not share the same number of nodes in its wave function. We also notice that the $e_{3}-h h_{3}$ has a slightly higher coupling strength compared with the other two symmetrical transitions. This difference corresponds to the fact that, compared to the 
other two hole wave functions, the $h h_{3}$ has its highest value at the center of the stack where the electromagnetic field is the strongest. The $e_{2}-h h_{2}$ has a lesser coupling strength because there is a node of the wave function at the antinode of the electromagnetic field.

Since our microcavity includes three stacks of QWs, the highest coupling strength for a single exciton state is [26] $\Omega_{0} / \sqrt{3}=2.31 \mathrm{meV}$. This value is smaller than the typical $3.5 \mathrm{meV}$ measured for single InGaAs QW in a $\lambda$ cavity $[17,22,24]$. This decrease of coupling strength is caused by three main factors: a decrease of the exciton binding energy which reduces the oscillator strength, a decrease of the overlap between the carrier wave function and the electromagnetic field, and an increase of the effective cavity length.

It is expected that the exciton binding energy in the QW stack should be less than its value in a single QW $(\sim 6-7 \mathrm{meV}$ for low indium QW $[38,39]$ ) because the confinement potential should decrease the strength of the Coulomb interaction. As shown in Fig. 2, the wave function extends over the full QW stack width corresponding to a thickness of $56 \mathrm{~nm}$. Compared with the usually assumed Bohr radius of about 10 to $12 \mathrm{~nm}$ for such III-V single QWs, [7] one concludes that the 2D approximation for the Coulomb interaction is no longer justified as the wave function extension along the confinement direction is much larger than the typical in-plane extension. As a result, the exciton Bohr radius should increase, leading to a reduction of the oscillator strength and therefore a decrease of the coupling strength.

The relative impact of the three factors affecting the coupling strength of the coupled QWs can be estimated by noting that $\Omega_{0} \propto L_{\mathrm{eff}}^{-\frac{1}{2}} F(0) \mu_{c v} I_{z}$, where $L_{\mathrm{eff}}=L_{\mathrm{cav}}+L_{\mathrm{DBR}}$ is the sum of the cavity spacer length and the penetration depth of the electromagnetic field inside the DBR, $F(0)$ is the exciton relative motion envelope wave function evaluated at its center, $\mu_{c v}$ the dipole matrix element, and $I_{z}$ the overlap integral as stated in Eq. (2) [40,41]. We define the ratio of the coupling strengths $\frac{\Omega_{c \mathrm{QW}}}{\Omega_{1 \mathrm{QW}}}=L^{-1 / 2} \cdot I \cdot F_{o s}^{1 / 2} \cdot I_{f z}^{-1 / 2}$, where $L^{-1 / 2}$ represent the ratio of the square root of the cavity effective lengths, $I$ is the ratio of the overlap integrals, $F_{o s}^{1 / 2}$ is the ratio of the square root of the oscillator strengths of the bare QW exciton, and $I_{f z}^{-1 / 2}$ is the square root of the inverse ratio of integrals of wave function overlap in the confinement direction without the cavity. This last term compensates the contribution of the overlap integral in $F_{o s}^{1 / 2}$, since it is taken into account in $I$. Calculation of the overlap integrals in both cases yields a ratio of $I \cong 0.90$. To estimate the influence of the effective cavity length, we note that, the penetration depth being of the order of $2 \mu \mathrm{m}$, an increase of the cavity spacer from $\lambda$ to $2 \lambda$ will decrease the coupling by a ratio of $L^{-1 / 2} \cong 0.95$. Combining these ratios and assuming $I_{f z}^{-1 / 2}=1$ gives $F_{o s}^{1 / 2} \cong$ 0.77 , meaning that the oscillator strength decreases by about $40 \%$ when passing from a single QW to a stack of three QWs and, consequently, decreases the coupling strength by $23 \%$.

Finally, we can achieve an even better fit of the measured polariton modes by adjusting the overlap integral manually. Doing so, all coupling strengths are slightly modified to get the best fit while keeping the parameter $\Omega_{0}$ unchanged. The result of this calculation is shown in Fig. 5(b) and the values of the integrals are given in Table I. The quadratic error is reasonably decreased to $\Delta E_{2}=4.66 \pm 0.02 \mathrm{meV}$, an improvement of $43 \%$. One sees a much better agreement of the fourth polariton line (the second brightest polariton mode in Fig. 4), and an excellent agreement within the experimental error bar. The highest coupling strength is now arising from the transition $e_{2}-h h_{2}$ instead of $e_{3}-h h_{3}$ as was found in the case of the first fitting procedure. The manual adjustment for the light-hole transition is justified since its wave function is not known. For the other transitions, the deviation between the calculated and adjusted coupling strengths indicates that the correction from the Coulomb interaction is not the same for each transition. Neglecting this interaction in our modeling is equivalent to assuming that all exciton transitions have the same binding energy and thus the same in-plane matrix element. Comparison between the bare QW measurements and the calculation (see Fig. 3) indicates indeed that this assumption is not justified. A calculation of the binding energy of each transition as well as possible state mixing (since some states share the same electron or hole) would be needed to properly explain the adjusted coupling strengths. Such calculation is beyond the scope of this study. Furthermore, it is likely that high index transitions are resonant with the continuum of the first transition. This interaction is expected to decrease further the coupling to the electromagnetic field and also broaden the transition, which might explain the linewidth and large coupling strength deviation obtained for the $e_{3}-h h_{3}$ exciton. Moreover, we expect the $e_{2}-h h_{2}$ transition to be less affected by coupling effect than the $e_{1}-h h_{1}$, the node at the center of the wave function making it more likely to find the exciton confined in the outer wells instead of being delocalized over the whole stack. This might also explain why the $e_{2}-h h_{2}$ transition becomes more strongly coupled to the cavity mode in the manual fit.

\section{CONCLUSIONS}

In this work, we studied the effect of the electronic coupling between the stacked QWs on the strong coupling in a planar microcavity. The QW electronic coupling is evidenced when comparing PL and PLE spectra of a bare QW stack with effective mass calculation. When the QW stack is placed inside the microcavity, the existence of a coupling between QWs causes two main effects: first an increase of the number of polariton modes and a decrease of the coupling strength compared to that of a single QW. The latter is interpreted as a decrease of the exciton binding energy and of the oscillator strength due to the delocalization of the exciton wave function over the QW stack. Using a coupled oscillator model accounting for the calculated electron-hole transitions, we simulated all measured polariton modes using a single coupling parameter. The deviation from this simple model is understood as an interplay between the Coulomb interaction and the extension of $e-h h$ wave function over the QW stack.

\section{ACKNOWLEDGMENTS}

The present work is supported by the Swiss National Science Foundation under Project N 153620, the Quantum Photonics National Center of Competence in research $\mathrm{N}$ 128792 and the European Research Council Project Polaritonics Contract N 291120. The Polatom network is also acknowledged. 
[1] J. J. Hopfield, Phys. Rev. 112, 1555 (1958).

[2] C. Weisbuch, M. Nishioka, A. Ishikawa, and Y. Arakawa, Phys. Rev. Lett. 69, 3314 (1992).

[3] J. Kasprzak, M. Richard, S. Kundermann, A. Baas, P. Jeambrun, J. M. J. Keeling, F. M. Marchetti, M. H. Szymanska, R. André, J. L. Staehli et al., Nature (London) 443, 409 (2006).

[4] E. Wertz, L. Ferrier, D. D. Solnyshkov, P. Senellart, D. Bajoni, A. Miard, A. Lemaître, G. Malpuech, and J. Bloch, Appl. Phys. Lett. 95, 051108 (2009).

[5] D. Bajoni, P. Senellart, E. Wertz, I. Sagnes, A. Miard, A. Lemaître, and J. Bloch, Phys. Rev. Lett. 100, 047401 (2008).

[6] G. Christmann, R. Butté, E. Feltin, J.-F. Carlin, and N. Grandjean, Appl. Phys. Lett. 93, 051102 (2008).

[7] R. Houdré, J. L. Gibernon, P. Pellandini, R. P. Stanley, U. Oesterle, C. Weisbuch, J. O'Gorman, B. Roycroft, and M. Ilegems, Phys. Rev. B 52, 7810 (1995).

[8] R. Butté, G. Delalleau, A. I. Tartakovskii, M. S. Skolnick, V. N. Astratov, J. J. Baumberg, G. Malpuech, A. Di Carlo, A. V. Kavokin, and J. S. Roberts, Phys. Rev. B 65, 205310 (2002).

[9] L. Kappei, J. Szczytko, F. Morier-Genoud, and B. Deveaud, Phys. Rev. Lett. 94, 147403 (2005).

[10] M. Moran, P. Dawson, and K. J. Moore, Solid State Commun. 107, 119 (1998).

[11] T. Wang, M. Bayer, A. Forchel, N. A. Gippius, and V. Kulakovskii, Phys. Rev. B 62, 7433 (2000).

[12] G. Nardin, G. Moody, R. Singh, T. M. Autry, H. Li, F. MorierGenoud, and S. T. Cundiff, Phys. Rev. Lett. 112, 046402 (2014).

[13] A. Armitage, M. S. Skolnick, A. V. Kavokin, D. M. Whittaker, V. N. Astratov, G. A. Gehring, and J. S. Roberts, Phys. Rev. B 58, 15367 (1998).

[14] G. Panzarini, L. C. Andreani, A. Armitage, D. Baxter, M. S. Skolnick, V. N. Astratov, J. S. Roberts, A. V. Kavokin, M. R. Vladimirova, and M. A. Kaliteevski, Phys. Rev. B 59, 5082 (1999).

[15] M. Emam-Ismail, V. N. Astratov, M. S. Skolnick, D. M. Whittaker, and J. S. Roberts, Phys. Rev. B 62, 1552 (2000).

[16] C. Diederichs and J. Tignon, Appl. Phys. Lett. 87, 251107 (2005).

[17] O. El Daif, A. Baas, T. Guillet, J.-P. Brantut, R. I. Kaitouni, J. L. Staehli, F. Morier-Genoud, and B. Deveaud, Appl. Phys. Lett. 88, 061105 (2006).

[18] H. Cao, S. Pau, Y. Yamamoto, and G. Björk, Phys. Rev. B 54, 8083 (1996).

[19] J. Wainstain, C. Delalande, D. Gendt, M. Voos, J. Bloch, V. Thierry-Mieg, and R. Planel, Phys. Rev. B 58, 7269 (1998).

[20] R. Rapaport, R. Harel, E. Cohen, A. Ron, E. Linder, and L. N. Pfeiffer, Phys. Rev. Lett. 84, 1607 (2000).
[21] M. Perrin, P. Senellart, A. Lemaître, and J. Bloch, Phys. Rev. B 72, 075340 (2005).

[22] G. R. Hayes, S. Haacke, M. Kauer, R. P. Stanley, R. Houdré, U. Oesterle, and B. Deveaud, Phys. Rev. B 58, R10175 (1998).

[23] R. Houdre, C. Weisbuch, R. P. Stanley, U. Oesterle, and M. Ilegems, Phys. Rev. Lett. 85, 2793 (2000).

[24] M. Saba, F. Quochi, C. Ciuti, U. Oesterle, J. L. Staehli, B. Deveaud, G. Bongiovanni, and A. Mura, Phys. Rev. Lett. 85, 385 (2000).

[25] R. Houdré, C. Weisbuch, R. P. Stanley, U. Oesterle, P. Pellandini, and M. Ilegems, Phys. Rev. Lett. 73, 2043 (1994).

[26] R. Houdré, R. P. Stanley, and M. Ilegems, Phys. Rev. A 53, 2711 (1996).

[27] G. Bongiovanni, A. Mura, F. Quochi, S. Gürtler, J. L. Staehli, F. Tassone, R. P. Stanley, U. Oesterle, and R. Houdré, Phys. Rev. B 55, 7084 (1997).

[28] A. I. Tartakovskii, M. Emam-Ismail, R. M. Stevenson, M. S. Skolnick, V. N. Astratov, D. M. Whittaker, J. J. Baumberg, and J. S. Roberts, Phys. Rev. B 62, R2283 (2000).

[29] R. M. Stevenson, V. N. Astratov, M. S. Skolnick, J. S. Roberts, and G. Hill, Phys. Rev. B 67, 081301 (2003).

[30] A. A. Khalifa, A. P. Love, D. N. Krizhanovskii, M. S. Skolnick, and J. S. Roberts, Appl. Phys. Lett. 92, 061107 (2008).

[31] www.nextnano.de.

[32] J. Y. Marzin, M. N. Charasse, and B. Sermage, Phys. Rev. B 31, 8298 (1985).

[33] G. Bastard, Wave Mechanics Applied to Semiconductor Heterostructures (Les éditions de physique, Les Ulis Cedex, 1988).

[34] J. Szczytko, L. Kappei, J. Berney, F. Morier-Genoud, M. T. Portella-Oberli, and B. Deveaud, Phys. Rev. B 71, 195313 (2005).

[35] B. Deveaud, J. Y. Emery, A. Chomette, B. Lambert, and M. Baudet, Appl. Phys. Lett. 45, 1078 (1984).

[36] P. Lugan, D. Sarchi, and V. Savona, Phys. Stat. Sol. C 3, 2428 (2006).

[37] M. Kira and S. W. Koch, Semiconductor Quantum Optics, 1st ed. (Cambridge University Press, Cambridge, 2012).

[38] H. Q. Hou, Y. Segawa, Y. Aoyagi, S. Namba, and J. M. Zhou, Phys. Rev. B 42, 1284 (1990).

[39] J. Szczytko, L. Kappei, J. Berney, F. Morier-Genoud, M. T. Portella-Oberli, and B. Deveaud, Phys. Rev. Lett. 93, 137401 (2004).

[40] V. Savona, Z. Hradil, A. Quattropani, and P. Schwendimann, Phys. Rev. B 49, 8774 (1994).

[41] V. Savona, L. C. Andreani, P. Schwendimann, and A. Quattropani, Solid State Commun. 93, 733 (1995). 